\title{
Population pharmacokinetic analysis of linezolid in patients with different types of shock: Effect of platelet count
}

\author{
DONGDONG WANG $^{1^{*}}$, XIAOFEI ZHENG ${ }^{2 *}$, YANG YANG $^{3}$ and XIAO CHEN ${ }^{4}$ \\ ${ }^{1}$ Department of Pharmacy, Children's Hospital of Fudan University, Shanghai 201102; \\ ${ }^{2}$ Department of Medical Imaging, Zhongda Hospital Affiliated to Southeast University, Nanjing, Jiangsu 210000; \\ ${ }^{3}$ Jiangsu Key Laboratory of New Drug Research and Clinical Pharmacy, Xuzhou Medical University, Xuzhou, \\ Jiangsu 221004; ${ }^{4}$ Department of Pharmacy, The People's Hospital of Jiangyin, Jiangyin, Jiangsu 214400, P.R. China
}

Received January 8, 2019; Accepted May 24, 2019

DOI: $10.3892 /$ etm.2019.7747

\begin{abstract}
Linezolid was approved by the Food and Drug Administration for the treatment of serious infections. However, patients with serious frequently develop shock, and it is currently elusive whether shock affects the pharmacokinetics of linezolid. The aim of the present study was to explore whether the pharmacokinetics of linezolid are different among patients with various types of shock or patients without shock and whether potential confounders are involved in their outcomes. A population pharmacokinetic analysis using a non-linear mixed-effects model was performed to examine the pharmacokinetics of patients with different types of shock or patients without shock. The pharmacokinetics of linezolid in patients with different types of shock or patients without shock was described by a one-compartment model. In our results, the patients with different types of shock or patients without shock demonstrated no differences in pharmacokinetics, whereas the platelet count was identified as a significant influencing factor. The results demonstrated that the pharmacokinetics of linezolid exhibited no significant differences among patients with different types of shock or patients without shock, whereas the platelet count significantly affected the clearance rate of linezolid.
\end{abstract}

\section{Introduction}

Linezolid is a unique synthetic antimicrobial agent of the oxazolidinone class of antibiotics with activity against all gram-positive microorganisms, including certain mycobacteria

Correspondence to: Miss Xiao Chen, Department of Pharmacy, The People's Hospital of Jiangyin, 163 Shoushan Road, Jiangyin, Jiangsu 214400, P.R. China

E-mail: chenxiao112733@163.com

*Contributed equally

Key words: linezolid, shock patients, population pharmacokinetics, platelet and various gram-negative anaerobes (1-4). This drug was approved by the Food and Drug Administration in April 2000 for the treatment of serious infections caused by gram-positive microorganisms, including those with known multidrug resistance, namely methicillin-resistant Staphylococcus aureus and vancomycin-resistant Enterococcus faecium (4). In addition, linezolid has been used as a second-line agent for treating tuberculosis (5-7) and exhibited no cross-resistance with other anti-tuberculosis drugs (8-10).

Pharmacokinetic studies of linezolid have been previously performed (11-13). It has been indicated that linezolid is used to treat patients with serious infections and in patients that develop frequent shock (14-17). To date, it has remained elusive whether the different types of shock affect the pharmacokinetic parameters of linezolid. In the present study, a population pharmacokinetic (PPK) analysis was performed to explore whether the pharmacokinetics of linezolid differ among patients with different types of shock or patients without shock and to explore the potential influencing factors.

\section{Methods}

Patients and data collection. The data used in the present study were obtained from clinical routine diagnostic examinations and treatments of shock patients treated between January 2016 and August 2018 at the Zhongda Hospital affiliated to Southeast University (Nanjing, China). The clinical information available from the database was retrospectively reviewed. The retrospective inclusion criteria were as follows: Subjects (patients without shock, septic shock, hemorrhagic shock, neurogenic shock and cardiogenic shock) treated with linezolid (600 mg linezolid every $12 \mathrm{~h}$, intravenously). Regarding the different types of shock, the patients were treated with different therapeutic regimens. In order to avoid the influence of drug interactions from different therapeutic schemes on linezolid pharmacokinetics, the following exclusion criteria were set: Combined use of drugs, which may have affected the pharmacokinetics of linezolid. Blood concentrations were obtained from the records of therapeutic drug monitoring. The associated clinical data were from medical log information. Prior to the present study, the data (blood parameters and medical log information) were already 
available, no organized collection or biological detection was required, and the information was retrospectively collated and analyzed. Using the already recorded and available blood concentrations and medical log information, the PPK model was built. The present study was approved by the Research Ethics Committee of the Zhongda Hospital affiliated to the Southeast University (Nanjing, China). The present study is a retrospective study and the analysis was approved by the affiliated ethics committee without any requirement for written informed consent.

The medical information included the following parameters: Sex, age, albumin (ALB), globulin (GLB), ALB/GLB (A/G), alanine transaminase (ALT), aspartate transaminase (AST), serum creatinine (SCR), urea, total protein (TP), total bile acid (TBA), total bilibrubin (TBIL), hematocrit (HCT), hemoglobin (HGB), mean corpuscular hemoglobin $(\mathrm{MCH})$ and mean corpuscular hemoglobin concentration (MCHC). In addition, the platelet count (PLT) was measured. Furthermore, the patient status (patients without shock, septic shock, hemorrhagic shock, neurogenic shock and cardiogenic shock) was also recorded.

PPK modeling. The data were analysed using the non-linear mixed-effects model (NONMEM) computer program (version VII; ICON Development Solutions). A one-compartment model with clearance (CL) and volumes of distribution (V) was used to describe the pharmacokinetic parameters of linezolid in the population of the present study.

Random-effects model. The inter-individual variabilities were evaluated by the exponential error model, according to the following equation:

$P_{i}=T(P) x \exp \left(\eta_{i}\right)(a)$, where $P_{i}$ is the individual parameter value, $T(P)$ is the typical individual parameter value and $\eta_{i}$ is the symmetrical distribution, which includes zero-mean chance variables with a variance.

The variabilities of the residual error variability were estimated as follows:

$\mathrm{OB}=\mathrm{IP} \times\left(1+\varepsilon_{1}\right)(\mathrm{b})$, where $\mathrm{OB}$ is the observation and IP represents the individual predicted concentration. $\varepsilon_{1}$ represents the symmetrical distribution, which includes zero-mean chance variables with a variance.

Covariate model. The following two equations describe the correlation of the parameters between continuous and categorical covariates, respectively.

$\mathrm{P}_{\mathrm{i}}=\mathrm{T}(\mathrm{P}) \times\left(\operatorname{Cov}_{\mathrm{i}} / \operatorname{Cov}_{\text {constant }}\right)^{\theta}(\mathrm{c}) \mathrm{P}_{\mathrm{i}}=\mathrm{T}(\mathrm{P}) \times\left(1+\theta \times \operatorname{Cov}_{\mathrm{i}}\right)(\mathrm{d})$, where $\mathrm{P}_{\mathrm{i}}$ is the individual parameter value and $\mathrm{T}(\mathrm{P})$ is the typical individual parameter value. $\theta$ is the estimated parameter and $\operatorname{Cov}_{i}$ is the covariate of the i-th individual. $\operatorname{Cov}_{\text {constant }}$ was fixed at a value similar to the population median of the covariate.

The potential covariates were sex, age, ALB, GLB, A/G, ALT, AST, SCR, UREA, TP, TBA, TBIL, PLT, HCT, HGB, $\mathrm{MCH}$ and MCHC levels, as well as the different types of shock. The stepwise-way set-up covariate model and likelihood ratio were used to compare the hierarchical models. The alterations in the objective function values (OFV) were produced by covariate inclusions and a decrease of OFV to $>3.84(\mathrm{P}<0.05)$ was deemed as the inclusion standard of the covariates into the basic model $(18,19)$. Following the establishment of a full regression model, the assessment was performed by deleting covariates from each parameter one by one in order to obtain the final model. An increase in OFV to $>6.64(\mathrm{P}<0.01)$ was considered as a standard for significant associations in the final model $(18,19)$.

Model validation. The final model was evaluated using bootstrap, an internal validation method, which was generated by repeated random sampling with replacement from the raw database. The process was performed by the software Wings for NONMEM, which performed 1,000 repetitions with different random sampling. The medians and percentiles (2.5-97.5) from bootstrap consequences were used for comparison of these values with those derived from the final model. Visual inspection of routine diagnostic plots included observations vs. individual predictions and absolute value of weighted residuals (iWRES) vs. individual predictions. The prediction-corrected visual predictive check (VPC) plots were used to assess the predictive performance of the final model.

\section{Results}

Data collection. The data of 37 Chinese patients treated with linezolid were analyzed, and their demographic and laboratory data are provided in Table I. The pharmacokinetic profiles of linezolid in patients with different types of shock are provided in Fig. 1A-E. The results revealed that the concentration-time relation from patients with different types of shock or patients without shock demonstrated certain differences. However, whether the differences were a result of patient status or other potential influencing factors requires further study in future research. The population included 18 patients without shock, 11 septic shock patients, 1 hemorrhagic shock patient, 4 neurogenic shock patients and 3 cardiogenic shock patients (Fig. 1F).

Modeling. All potential covariates were analysed in the present study, and only the PLT on CL covariate exhibited statistical significance. The changes in the OFV are presented in Table II and the final model was constructed as follows:

$\mathrm{CL}=\theta_{\mathrm{CL}} \times(\mathrm{PLT} / 200)^{\wedge} \theta_{\mathrm{PLT}} \times \omega_{\mathrm{CL}}(\mathrm{e}) \mathrm{V}=\theta_{\mathrm{V}} \times \omega_{\mathrm{V}}$ (f), where $\mathrm{CL}, \mathrm{V}, \theta_{\mathrm{CL}}, \theta_{\mathrm{V},}, \theta_{\mathrm{PLT}}, \omega_{\mathrm{CL}}$ and $\omega_{\mathrm{V}}$ are the clearance, volume of distribution, typical value of $\mathrm{CL}$, typical value of $\mathrm{V}$, the coefficient of the platelet, inter-individual variability of CL and inter-individual variability of $\mathrm{V}$, respectively. The platelet count was included in the covariates and the clearance rate of linezolid was increased in parallel with an increase in the platelet count.

Validation. The routine diagnostic plots that were visually inspected are provided in Fig. 2. They included the following comparison of the variables: Observations vs. individual predictions and iWRES vs. individual predictions. The parameter estimates in the final model and internal validation are provided in Table III. From 1,000 bootstrap runs, 992 runs were minimised with a successful covariance step and were included in the bootstrap analysis. The bootstrap median values were approximate to the estimate values in the final model, and the absolute value of all bias was $<6 \%$, indicating 
Table I. Demographic and laboratory data of the patients $(n=37)$.

\begin{tabular}{lcc}
\hline Characteristic & Mean \pm SD & Median (range) \\
\hline Sex (male/female) & $27 / 10$ & $/$ \\
Age (years) & $59.49 \pm 16.25$ & $62.00(29.00-89.00)$ \\
Albumin $(\mathrm{g} / \mathrm{l})$ & $32.57 \pm 3.40$ & $32.40(25.40-43.80)$ \\
Globulin $(\mathrm{g} / \mathrm{l})$ & $30.50 \pm 5.87$ & $30.10(15.40-40.40)$ \\
Albumin/globulin & $1.12 \pm 0.34$ & $1.01(0.67-2.30)$ \\
Alanine transaminase (IU/l) & $69.43 \pm 85.99$ & $48.00(3.00-525.00)$ \\
Aspartate transaminase $(\mathrm{IU} / \mathrm{l})$ & $57.89 \pm 41.92$ & $41.00(11.00-181.00)$ \\
Serum creatinine $(\mu$ mol/l) & $126.03 \pm 113.64$ & $85.00(16.00-499.00)$ \\
Urea (mmol/l) & $12.03 \pm 6.30$ & $11.10(2.40-29.90)$ \\
Total protein $(\mathrm{g} / \mathrm{l})$ & $62.76 \pm 7.00$ & $62.80(45.90-75.10)$ \\
Total bile acid $(\mu$ mol/l) & $7.20 \pm 13.06$ & $3.80(1.00-81.50)$ \\
Total bilibrubin $(\mu$ mol/l) & $25.04 \pm 65.96$ & $11.10(2.00-414.70)$ \\
Platelets $(10 / /)$ & $246.54 \pm 187.20$ & $213.00(11.00-895.00)$ \\
Hematocrit $(\%)$ & $27.14 \pm 5.32$ & $26.50(19.00-44.80)$ \\
Hemoglobin $(\mathrm{g} / \mathrm{l})$ & $89.84 \pm 17.68$ & $87.00(63.00-140.00)$ \\
Mean corpuscular hemoglobin $(\mathrm{pg})$ & $29.78 \pm 1.93$ & $29.90(25.30-35.00)$ \\
Mean corpuscular hemoglobin concentration $(\mathrm{g} / \mathrm{l})$ & $321.84 \pm 16.45$ & $321.00(277.00-355.00)$
\end{tabular}

SD, standard deviation.
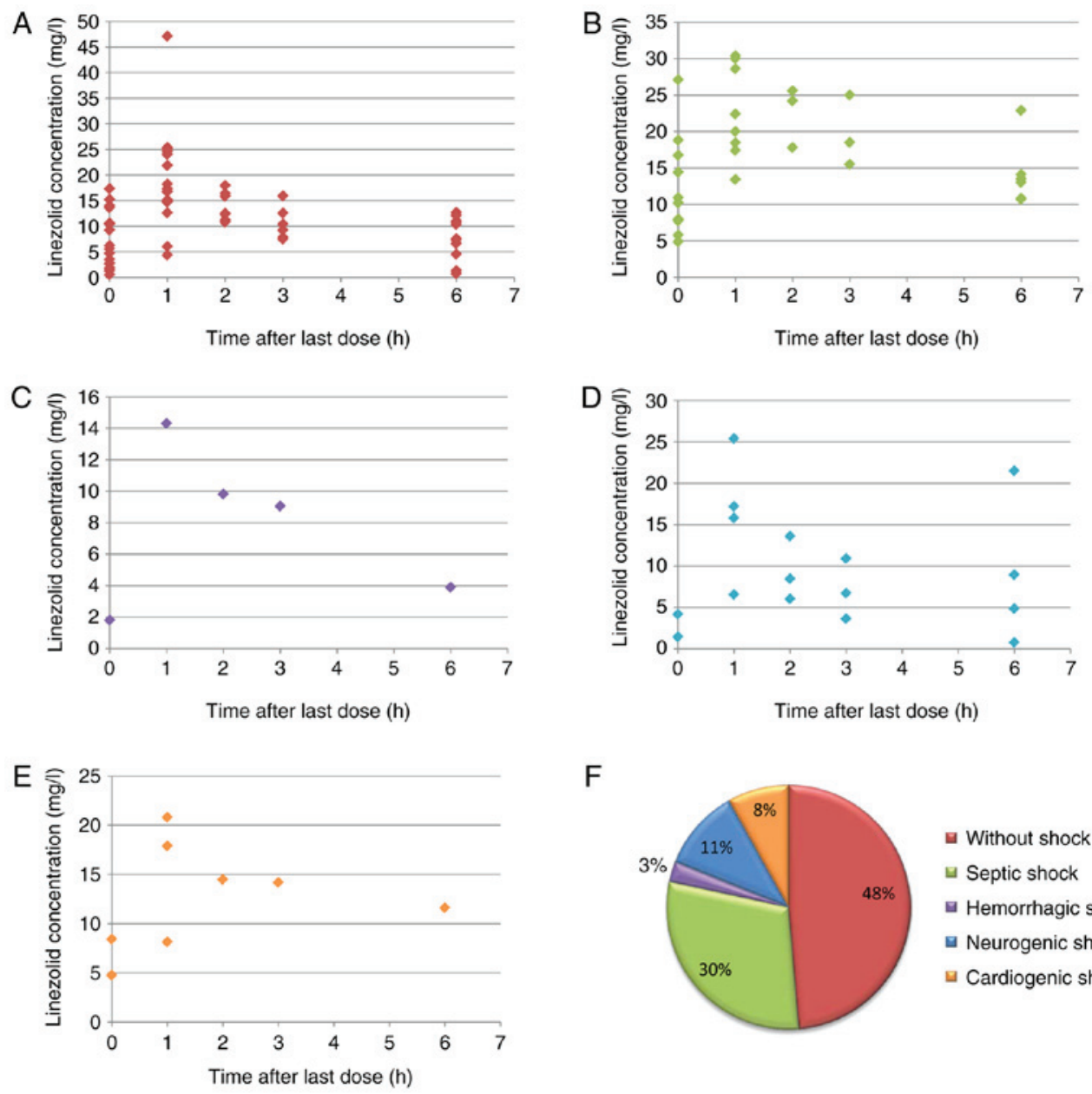

$\mathrm{F}$

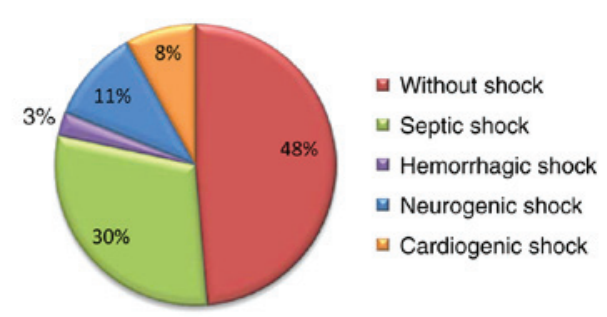

Figure 1. (A-E) Plasma Linezolid concentration in different types of shock patient following the last administration. (A) Patients without shock, (B) patients with septic shock, (C) patients with hemorrhagic shock, (D) patients with neurogenic shock and (E) patients with cardiogenic shock. (F) Constituent ratio of different types of shock patient. 
Table II. Change of objective function value of covariate analysis.

\begin{tabular}{|c|c|c|c|}
\hline Model description & $\mathrm{OFV}$ & $\triangle \mathrm{OFV}$ & P-value \\
\hline \multicolumn{4}{|l|}{ Inclusion step 1} \\
\hline Basic model & 1080.738 & / & / \\
\hline Influence of sex on CL & 1075.476 & -5.262 & $<0.05$ \\
\hline Influence of age on CL & 1079.979 & -0.759 & $>0.05$ \\
\hline Influence of absence of shock on CL & 1080.620 & -0.118 & $>0.05$ \\
\hline Influence of septic shock on CL & 1078.700 & -2.038 & $>0.05$ \\
\hline Influence of hemorrhagic shock on CL & 1079.133 & -1.605 & $>0.05$ \\
\hline Influence of neurogenic shock on CL & 1080.613 & -0.125 & $>0.05$ \\
\hline Influence of cardiogenic shock on CL & 1080.393 & -0.345 & $>0.05$ \\
\hline Influence of ALB on CL & 1078.277 & -2.461 & $>0.05$ \\
\hline Influence of GLB on CL & 1080.727 & -0.011 & $>0.05$ \\
\hline Influence of $\mathrm{A} / \mathrm{G}$ on $\mathrm{CL}$ & 1080.354 & -0.384 & $>0.05$ \\
\hline Influence of ALT on CL & 1080.723 & -0.015 & $>0.05$ \\
\hline Influence of AST on CL & 1080.719 & -0.019 & $>0.05$ \\
\hline Influence of SCR on CL & 1080.727 & -0.011 & $>0.05$ \\
\hline Influence of urea on CL & 1080.508 & -0.230 & $>0.05$ \\
\hline Influence of TP on CL & 1080.678 & -0.060 & $>0.05$ \\
\hline Influence of TBA on CL & 1077.249 & -3.489 & $>0.05$ \\
\hline Influence of TBIL on CL & 1077.598 & -3.140 & $>0.05$ \\
\hline Influence of PLT on CL & 1069.637 & -11.101 & $<0.05$ \\
\hline Influence of HCT on CL & 1079.774 & -0.964 & $>0.05$ \\
\hline Influence of HGB on CL & 1080.241 & -0.497 & $>0.05$ \\
\hline Influence of $\mathrm{MCH}$ on CL & 1077.168 & -3.570 & $>0.05$ \\
\hline Influence of $\mathrm{MCHC}$ on $\mathrm{CL}$ & 1080.212 & -0.526 & $>0.05$ \\
\hline \multicolumn{4}{|l|}{ Inclusion step 2} \\
\hline Influence of PLT on CL & 1069.637 & l & l \\
\hline Influence of PLT and sex on CL & 1067.456 & -2.181 & $>0.05$ \\
\hline \multicolumn{4}{|l|}{ Elimination } \\
\hline Full model & 1069.637 & / & l \\
\hline Elimination of PLT on CL & 1080.738 & 11.101 & $<0.01$ \\
\hline
\end{tabular}

OFV, objective function values; CL, clearance; ALB, albumin; GLB, globulin; A/G, ALB/GLB; ALT, alanine transaminase; AST, aspartate transaminase; SCR, serum creatinine; TP, total protein; TBA, total bile acid; TBIL, total bilibrubin; PLT, platelets; HCT, hematocrit; HGB, hemoglobin; MCH, mean corpuscular hemoglobin; MCHC, mean corpuscular hemoglobin concentration.

that the final model was dependent on specific parameters. The VPC plots for the final model are presented in Fig. 3, revealing that the most frequently observed concentration data were included in the $95 \%$ prediction intervals produced by the simulation data. Overall, the data suggested that the final model was able to predict drug concentrations with optimal efficiency.

\section{Discussion}

Linezolid is currently used for the treatment of severe infections caused by gram-positive microorganisms, including vancomycin-resistant Enterococcus, vancomycin-sensitive Enterococcus and Staphylococcus infections (20-22). Since linezolid has a time-dependent activity, the percentage of time during which plasma concentrations exceed the minimal inhibitory concentration (MIC) represents its efficacy.
Furthermore, the area under the concentration-time curve (AUC) over $24 \mathrm{~h}$ may be divided by the MIC ( $\left.\mathrm{AUC}_{0-24} / \mathrm{MIC}\right)$ and used for evaluating the pharmacokinetics of linezolid (23). A higher success rate was reported when plasma concentrations remained above the MIC for the entire dosing interval and when the $\mathrm{AUC}_{0-24} / \mathrm{MIC}$ values were between 80 and $120(24,25)$. This showcases the importance of studying the pharmacokinetics of linezolid for evaluating its efficacy. However, severe infections are frequently accompanied by shock (14-17), and whether different types of shock affect the pharmacokinetics of linezolid has remained elusive.

The application of PPK may provide useful information from limited data of patients. Furthermore, PPK analysis differentiates between inter-individual and intra-individual variabilities. Therefore, PPK is more reliable for confirming the effects of various factors with regard to pharmacokinetic parameters compared with the traditional use of 
Table III. Parameter estimates of final model and bootstrap validation.

\begin{tabular}{lccccc}
\hline & & & \multicolumn{2}{c}{ Bootstrap (n=992) } & \\
\cline { 5 - 6 } Parameter & Estimate & SE & Median & $95 \% \mathrm{CI}$ & Bias (\%) \\
\hline $\mathrm{CL}(\mathrm{l} / \mathrm{h})$ & 11.8 & 0.23 & 11.2 & {$[3.110,16.625]$} & -5.085 \\
$\mathrm{~V}(\mathrm{l})$ & 209 & 0.20 & 197 & {$[53.850,308.000]$} & -5.742 \\
$\theta_{\mathrm{PLT}}$ & 0.261 & 0.33 & 0.254 & {$[0.052,0.425]$} & -2.682 \\
$\omega_{\mathrm{CL}}$ & 0.299 & 2.15 & 0.287 & {$[0.212,0.359]$} & -4.013 \\
$\omega_{\mathrm{V}}$ & 0.299 & 2.15 & 0.287 & {$[0.211,0.356]$} & -4.013 \\
$\sigma_{1}$ & 1.020 & 0.08 & 1.015 & {$[0.864,1.179]$} & -0.490 \\
\hline
\end{tabular}

95\% CI is displayed as the 2.5th, 97.5th percentile of bootstrap estimates. CL, clearance; V, volume of distribution; $\theta_{\mathrm{PLT}}$, was the coefficient of the platelet; $\omega_{\mathrm{CL}}$, inter-individual variability of $\mathrm{CL} ; \omega_{\mathrm{V}}$, inter-individual variability of $\mathrm{V}$; $\sigma_{1}$, residual variability (proportional error); bias, prediction error $[$ Bias $=($ Median-Estimate $) /$ Estimate $\mathrm{x} 100 \%]$.
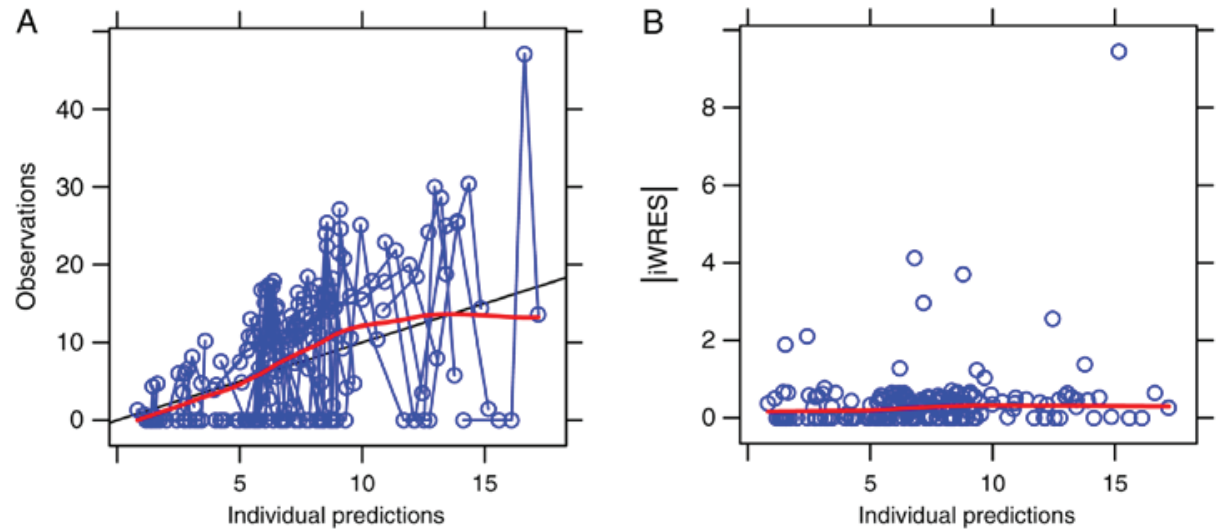

Figure 2. Visual inspection of routine diagnostic plots of the final population model. (A) Observations vs. individual predictions. (B) Absolute value of iWRES vs. individual predictions. iWRES, weighted residuals.

pharmacokinetics $(18,19,26-33)$. In the present study, it was investigated whether the pharmacokinetics of linezolid are different in patients with different types of shock or patients without shock and the effects of several potential confounders on its metabolic profile were assessed. Among these groups, the population characteristics and biological features were screened as covariates.

In the present study, the pharmacokinetics of linezolid in patients with different types of shock or patients without shock was described by a one-compartment model with the parameters $\mathrm{CL}$ and $\mathrm{V}$. The typical values of $\mathrm{CL}$ and $\mathrm{V}$ in the final PPK model were $11.8 \mathrm{l} / \mathrm{h}$ and 209 liters, respectively. Plock et al (34) reported on the population pharmacokinetics of linezolid of 10 healthy volunteers and 24 septic patients and the typical value of CL was estimated to be $11.1 \mathrm{l} / \mathrm{h}$, which was similar to the results obtained in the present study. Of note, the present study determined that patients with different types of shock or patients without shock exhibited no differences in pharmacokinetics, suggesting that patient status (patients without shock, septic shock, hemorrhagic shock, neurogenic shock and cardiogenic shock) was not an influencing factor. However, when the platelet count was included in the covariates, the clearance rate of linezolid increased with the increase of the platelet

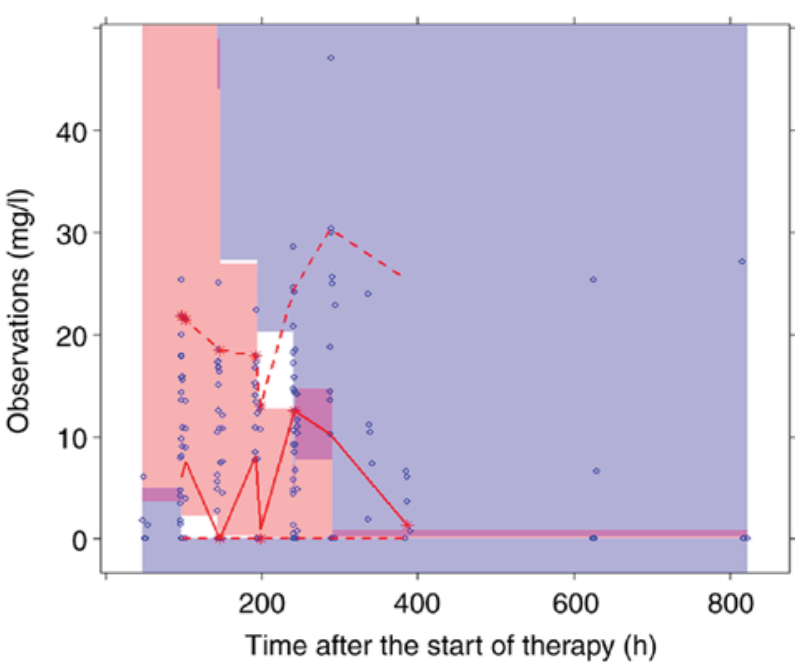

Figure 3. Prediction-corrected visual predictive check for the final model. The middle solid line represents the median of the prediction-corrected concentrations. The lower and upper dashed lines represent the 2.5 and 97.5 th percentiles of the prediction-corrected concentrations, respectively.

count. Therefore, the data suggested that adjustment of a patient's linezolid regimen is possible, provided that the 
platelet count is measured and the linezolid concentration values are adjusted accordingly.

The present study has a limitation: The analysis and patient inclusion were performed at a single center. Therefore, further multicenter and prospective studies with a larger number of patients are required.

In conclusion, the pharmacokinetics of linezolid were not different among patients without shock, as well as patients with septic shock, hemorrhagic shock, neurogenic shock and cardiogenic shock. However, the platelet count significantly influenced the clearance rate of linezolid.

\section{Acknowledgements}

Not applicable.

\section{Funding}

The current study was supported by AOSAIKANG pharmaceutical foundation (grant no. A201826), The Young Medical Talents of Wuxi (grant no. QNRC020), Young Project of Wuxi Health and Family Planning Research (grant no. Q201706) and the Wuxi Science and Technology Development Guidance Plan (medical and health care; grant no. CSZON1744).

\section{Availability of data and materials}

The datasets used and/or analysed during the present study are available from the corresponding author on reasonable request.

\section{Authors' contributions}

$\mathrm{XC}$ and DW conceived and designed the study. XZ collected the data. DW, XZ and YY built the model. DW wrote the manuscript. XZ and YY reviewed and edited the manuscript. All authors read and approved the final manuscript.

\section{Ethics approval and consent to participate}

The present study was approved by the Research Ethics Committee of the Zhongda Hospital affiliated to Southeast University (Nanjing, China). The retrospective analysis was approved by the ethics committee without the requirement for written informed consent, since the data were collected without patient identifiers.

\section{Patient consent for publication}

Not applicable.

\section{Competing interests}

The authors declare that they have no competing interests.

\section{References}

1. Ashtekar DR, Costa-Periera R, Shrinivasan T, Iyyer R, Vishvanathan $\mathrm{N}$ and Rittel W: Oxazolidinones, a new class of synthetic antituberculosis agent. In vitro and in vivo activities of DuP-721 against Mycobacterium tuberculosis. Diagn Microbiol Infect Dis 14: 465-471, 1991
2. Jones RN, Johnson DM and Erwin ME: In vitro antimicrobial activities and spectra of U-100592 and U-100766, two novel fluorinated oxazolidinones. Antimicrob Agents Chemother 40: 720-726, 1996.

3. Kaatz GW and Seo SM: In vitro activities of oxazolidinone compounds U100592 and U100766 against Staphylococcus aureus and Staphylococcus epidermidis. Antimicrob Agents Chemother 40: 799-801, 1996.

4. Meagher AK, Forrest A, Rayner CR, Birmingham MC and Schentag JJ: Population pharmacokinetics of linezolid in patients treated in a compassionate-use program. Antimicrob Agents Chemother 47: 548-553, 2003.

5. Condos R, Hadgiangelis N, Leibert E, Jacquette G, Harkin T and Rom WN: Case series report of a linezolid-containing regimen for extensively drug-resistant tuberculosis. Chest 134: 187-192, 2008.

6. Fortun J, Martin-Davila P, Navas E, Perez-Elias MJ, Cobo J, Tato M, De la Pedrosa EG, Gomez-Mampaso E and Moreno S: Linezolid for the treatment of multidrug-resistant tuberculosis. J Antimicrob Chemother 56: 180-185, 2005.

7. Park IN, Hong SB, Oh YM, Kim MN, Lim CM, Lee SD, Koh Y, Kim WS, Kim DS, Kim WD and Shim TS: Efficacy and tolerability of daily-half dose linezolid in patients with intractable multidrug-resistant tuberculosis. J Antimicrob Chemother 58: 701-704, 2006.

8. Cynamon MH, Klemens SP, Sharpe CA and Chase S: Activities of several novel oxazolidinones against mycobacterium tuberculosis in a murine model. Antimicrob Agents Chemother 43: 1189-1191, 1999

9. Molicotti P, Ortu S, Bua A, Cannas S, Sechi LA and Zanetti S: In vitro efficacy of Linezolid on clinical strains of Mycobacterium tuberculosis and other mycobacteria. New Microbiol 29: 275-280, 2006.

10. Sood R, Bhadauriya T, Rao M, Gautam R, Malhotra S,Barman TK, Upadhyay DJ and Rattan A: Antimycobacterial activities of oxazolidinones: A review. Infect Disord Drug Targets 6: 343-354, 2006.

11. Keel RA, Schaeftlein A, Kloft C, Pope JS, Knauft RF, Muhlebach M, Nicolau DP and Kuti JL: Pharmacokinetics of intravenous and oral linezolid in adults with cystic fibrosis. Antimicrob Agents Chemother 55: 3393-3398, 2011.

12. Sazdanovic P, Jankovic SM, Kostic M, Dimitrijevic A and Stefanovic S: Pharmacokinetics of linezolid in critically ill patients. Expert Opin Drug Metab Toxicol 12: 595-600, 2016.

13. Buerger C, Plock N, Dehghanyar P, Joukhadar C and Kloft C: Pharmacokinetics of unbound linezolid in plasma and tissue interstitium of critically ill patients after multiple dosing using microdialysis. Antimicrob Agents Chemother 50: 2455-2463, 2006.

14. Calzada Y, Jordan I, Vila-Perez D, Cambra FJ and MunozAlmagro C: Pleuropneumonia and septic shock due to multiresistant Streptococcus pneumoniae serotype 19A treated with linezolid. An Pediatr (Barc) 81: e22-23, 2014 (In Spanish).

15. De Bels D, Garcia-Filoso A, Jeanmaire M, Preseau T, Miendje Deyi VY and Devriendt J: Successful treatment with linezolid of septic shock secondary to methicillin-resistant Staphylococcus aureus arthritis. J Antimicrob Chemother 55: 812-813, 2005.

16. Rac H, Bojikian KD, Lucar J and Barber KE: Successful treatment of necrotizing fasciitis and streptococcal toxic shock syndrome with the addition of linezolid. Case Rep Infect Dis 2017: 5720708, 2017.

17. Stevens DL, Wallace RJ, Hamilton SM and Bryant AE: Successful treatment of staphylococcal toxic shock syndrome with linezolid: A case report and in vitro evaluation of the production of toxic shock syndrome toxin type 1 in the presence of antibiotics. Clin Infect Dis 42: 729-730, 2006.

18. Wang DD, Lu JM, Li Q and Li ZP: Population pharmacokinetics of tacrolimus in paediatric systemic lupus erythematosus based on real-world study. J Clin Pharm Ther 43: 476-483, 2018.

19. Vadcharavivad S, Praisuwan S, Techawathanawanna N, Treyaprasert W and Avihingsanon Y: Population pharmacokinetics of tacrolimus in Thai kidney transplant patients: Comparison with similar data from other populations. J Clin Pharm Ther 41: 310-328, 2016.

20. Sahuquillo Arce JM, Colombo Gainza E, Gil Brusola A, Ortiz Estevez R, Canton E and Gobernado M: In vitro activity of linezolid in combination with doxycycline, fosfomycin, levofloxacin, rifampicin and vancomycin against methicillin-susceptible Staphylococcus aureus. Rev Esp Quimioter 19: 252-257, 2006. 
21. Sweeney MT and Zurenko GE: In vitro activities of linezolid combined with other antimicrobial agents against Staphylococci, Enterococci, Pneumococci, and selected gram-negative organisms. Antimicrob Agents Chemother 47: 1902-1906, 2003.

22. Beitdaghar M, Ahmadrajabi R, Karmostaji A and Saffari F: In vitro activity of linezolid alone and combined with other antibiotics against clinical enterococcal isolates. Wien Med Wochenschr 169: 215-221, 2019.

23. Craig WA: Basic pharmacodynamics of antibacterials with clinical applications to the use of beta-lactams, glycopeptides, and linezolid. Infect Dis Clin North Am 17: 479-501, 2003.

24. Roger C, Muller L, Wallis SC, Louart B, Saissi G, Lipman J, Lefrant JY and Roberts JA: Population pharmacokinetics of linezolid in critically ill patients on renal replacement therapy: Comparison of equal doses in continuous venovenous haemofiltration and continuous venovenous haemodiafiltration. J Antimicrob Chemother 71: 464-470, 2016.

25. Rayner CR, Forrest A, Meagher AK, Birmingham MC and Schentag JJ: Clinical pharmacodynamics of linezolid in seriously ill patients treated in a compassionate use programme. Clin Pharmacokinet 42: 1411-1423, 2003.

26. Wang DD, Lu JM, Li YZ, Li Q and Li ZP: Population pharmacokinetics of sirolimus in pediatric tuberous sclerosis complex: From real world study. Int J Clin Exp Med 11: 12302-12309, 2018.

27. Chen Y, Wu D, Dong M, Zhu Y, Lu J, Li X, Chen C and Li Z: Population pharmacokinetics of vancomycin and AUC-guided dosing in Chinese neonates and young infants. Eur J Clin Pharmacol 74: 921-930, 2018.

28. Gong Y, Chen Y, Li Q and Li Z: Population pharmacokinetic analysis of digoxin in Chinese neonates and infants. J Pharmacol Sci 125: 142-149, 2014.
29. Li Z, Chen Y, Li Q, Cao D, Shi W, Cao Y, Wu D, Zhu Y, Wang Y and Chen C: Population pharmacokinetics of piperacillin/tazobactam in neonates and young infants. Eur J Clin Pharmacol 69: 1223-1233, 2013

30. Andrews LM, Hesselink DA, van Gelder T, Koch BC, Cornelissen EA, Bruggemann RJ, van Schaik RH, de Wildt SN, Cransberg K and de Winter BC: A population pharmacokinetic model to predict the individual starting dose of tacrolimus following pediatric renal transplantation. Clin Pharmacokinet 57: 475-489, 2018.

31. Yu ZC, Zhou PJ, Wang XH, Francoise B, Xu D, Zhang WX and Chen B: Population pharmacokinetics and Bayesian estimation of mycophenolic acid concentrations in Chinese adult renal transplant recipients. Acta Pharmacol Sin 38: 1566-1579, 2017.

32. Chen B, Shi HQ, Liu XX, Zhang WX, Lu JQ, Xu BM and Chen H Population pharmacokinetics and Bayesian estimation of tacrolimus exposure in Chinese liver transplant patients. J Clin Pharm Ther 42: 679-688, 2017

33. Chevillard L, Sabo N, Tod M, Labat L, Chasport C, Chevaleyre C, Thibaut F, Barre J, Azuar J, Questel F, et al: Population pharmacokinetics of oral baclofen at steady-state in alcoholic-dependent adult patients. Fundam Clin Pharmacol 32: 239-248, 2018.

34. Plock N, Buerger C, Joukhadar C, Kljucar S and Kloft C: Does linezolid inhibit its own metabolism? Population pharmacokinetics as a tool to explain the observed nonlinearity in both healthy volunteers and septic patients. Drug Metab Dispos 35: 1816-1823, 2007. Attribution-NonCommercial-NoDerivatives 4.0 International (CC BY-NC-ND 4.0) License. 